\title{
Grado de alfabetización académica en profesores de la Escuela de Nutrición de la Universidad Regional del Sureste
}

\author{
Degree of Academic Literacy in Teachers of the Universidad Regional del \\ Sureste
}

\section{Grau de alfabetização acadêmica em professores da Escola de Nutrição da Universidade Regional do Sudeste}

\author{
Enrique Salvador Neri Caballero \\ Universidad Regional del Sureste, Escuela de Nutrición, México \\ enrique.ner@profesores.urse.edu.mx \\ https://orcid.org/0000-0002-4896-0823
}

\section{Resumen}

El presente proyecto tiene como objetivo indagar el grado de conocimientos de los profesores de la Escuela de Nutrición de la Universidad Regional del Sureste en lo que se refiere a los lineamientos normativos más comunes en la producción de textos académicos. Se trata de una investigación de tipo descriptiva, con un diseño observacional-transversal. La muestra estuvo conformada por docentes de la Escuela de Nutrición. Asimismo, el presente estudio es de causalidad, con carácter dicotómico, es decir, propone una variable dependiente. conformada por los docentes de la Escuela de Nutrición, y una variable independiente, formulada como "Grado de alfabetización académica en los profesores de la Escuela de Nutrición”. Entre los resultados destaca que las normas de la American Psychological Association (APA) cuentan con un mayor alcance dentro del campo de la investigación y producción de textos académicos, debido a la mayor estructuración, aplicación y difusión que le dan a su normatividad. Se concluye que los profesores de la Escuela de Nutrición conocen los diferentes tipos de textos académicos (ensayos, relatorías y las monografías) y 


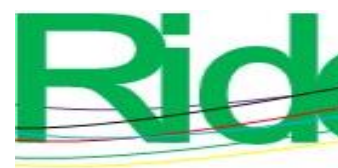

Revista Iberoamericana para la Investigación y el Desarrollo Educativo ISSN $2007-7467$

que, al igual que con los docentes, la mayor normativa seguida en la elaboración de trabajos escolares por parte de los estudiantes es la de la APA. Finalmente, se propone la elaboración de un manual normativo de la producción de textos académicos interno que brinde homogeneidad a los documentos que producen los estudiantes en las diferentes asignaturas que cursan a lo largo de su carrera.

Palabras clave: APA, docencia, MLA, nutrición, textos académicos.

\section{Abstract}

The objective of this project is to investigate the degree of knowledge of the professors of the School of Nutrition of the Universidad Regional del Sureste regarding the most common normative guidelines in the production of academic texts. It is a descriptive research, with an observational-cross-sectional design. The sample was made up of teachers from the School of Nutrition. Likewise, the present study is of causality, with a dichotomous character, that is, it proposes a dependent variable, the teachers of the School of Nutrition, and an independent variable, formulated as "Degree of academic literacy in the teachers of the School of Nutrition". Among the results, it stands out that the standards of the American Psychological Association (APA) have a greater reach within the field of research and production of academic texts, due to the greater structuring, application and dissemination that they give to their regulations. It is concluded that the teachers of the School of Nutrition know the different types of academic texts (essays, reports and monographs) and that, as with the teachers, the greater regulations followed in the preparation of school work by students is that of the APA. Finally, the development of a normative manual for the production of internal academic texts is proposed that provides homogeneity to the documents produced by students in the different subjects they study throughout their career.

Keywords: APA, teaching, MLA, nutrition, academic texts. 


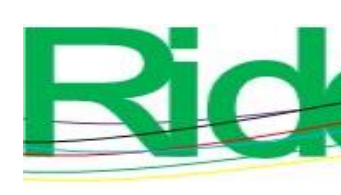

Revista Iberoamericana para la Investigación y el Desarrollo Educativo ISSN $2007-7467$

\section{Resumo}

O objetivo deste projeto é investigar o grau de conhecimento dos professores da Escola de Nutrição da Universidade Regional do Sudeste sobre as diretrizes normativas mais comuns na produção de textos acadêmicos. Trata-se de uma pesquisa descritiva, com delineamento observacional transversal. A amostra foi composta por professores da Escola de Nutrição. Da mesma forma, o presente estudo é de causalidade, de caráter dicotômico, ou seja, propõe uma variável dependente. composta pelos professores da Escola de Nutrição, e uma variável independente, formulada como "Grau de alfabetização acadêmica nos professores da Escola de Nutrição". Dentre os resultados, destaca-se que as normas da American Psychological Association (APA) têm maior abrangência no campo da pesquisa e produção de textos acadêmicos, devido à maior estruturação, aplicação e divulgação que conferem às suas regulamentações. Conclui-se que os professores da Escola de Nutrição conhecem os diferentes tipos de textos acadêmicos (ensaios, relatórios e monografias) e que, assim como os professores, a maior regulamentação seguida na elaboração dos trabalhos escolares pelos alunos é a da APA . Por fim, propõe-se o desenvolvimento de um manual normativo para a produção de textos acadêmicos internos que dê homogeneidade aos documentos produzidos pelos alunos nas diferentes disciplinas que estudam ao longo de sua carreira.

Palavras-chave: APA, ensino, MLA, nutrição, textos acadêmicos.

Fecha Recepción: Diciembre 2020

Fecha Aceptación: Julio 2021

\section{Introducción}

En la educación superior se presupone que la alfabetización académica de los estudiantes ya fue abordada en niveles escolares anteriores, como secundaria y preparatoria, por lo que el reforzamiento de esta habilidad queda relegada a la toma de cursos propedéuticos, talleres, tutorías, etc. De hecho, estudios confirman que es poco frecuente que los profesores del nivel educativo en cuestión destinen parte de su clase a la realización de lecturas dirigidas o a la elaboración de escritos en tiempo real o presente, y mucho menos al análisis de los productos de aprendizaje de ambas acciones. También dichos estudios de investigación, los cuales consistieron en comparar grupos donde se escribía y leía en el momento de la clase contra grupos en los que no, demostraron que los estudiantes de los que sí lo hacían terminaban con una comprensión del tema o contenido más solventada y, lo que 


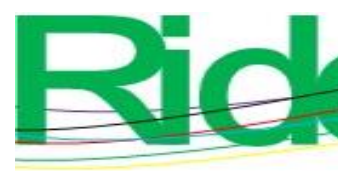

Revista Iberoamericana para la Investigación y el Desarrollo Educativo ISSN 2007 - 7467

es mejor en el aprendizaje, con una memoria a largo plazo del contenido (Carlino, 2017, p. 16).

El término de alfabetización académica suele confundirse con las habilidades de lectura y escritura que se obtienen al inicio de la vida escolar, estas últimas aprendidas y ejercitadas en un periodo de por lo menos nueve años en la estructura educativa mexicana. Las adquisiciones de estas habilidades iniciales corresponden a un proceso de alfabetización básico de la vida escolar de cualquier estudiante de este nivel. Cabe señalar que el concepto alfabetización suele ser antepuesto a otros provenientes de diferentes ramas o líneas del conocimiento, por ejemplo, la formación tecnológica (alfabetización tecnológica) o informática (alfabetización informática). Otros ejemplos como los anteriores son la alfabetización permanente o avanzada, que sugiere habilidades de orden más complicado para su adquisición. Ahora bien, volviendo a la alfabetización básica o inicial, es fácil percibir que es insuficiente o, al menos, que no cuenta con el tiempo necesario para cubrir todos los requerimientos que exige la producción de textos académicos de calidad. En consecuencia, podemos deducir que la alfabetización académica nos encamina a visualizar la suma de dificultades que se presentan en la producción de un texto académico, lo que permite poder contar con un enfoque más amplio de las correcciones necesarias que habrán de realizarse, a fin de obtener un producto de calidad de este tipo (Marín, 2006, p. 32).

El presente proyecto de investigación tiene como objetivo general indagar el grado de conocimientos de los profesores de la Escuela de Nutrición de la Universidad Regional del Sureste en lo que se refiere a los lineamientos normativos más comunes en la producción de textos académicos. Mientras que los objetivos específicos propuestos expresan la necesidad de investigar los lineamientos normativos de mayor uso en los profesores, así como averiguar cuántos tipos de textos académicos conocen y usan en su práctica docente. La justificación de emprender este tipo de pesquisas estriba en conocer las herramientas de las que echa mano el profesor tanto en la producción de textos personales como en los lineamientos de redacción académica que propone a sus alumnos. El planteamiento del problema de la presente investigación surge de la percepción de que cada profesor del claustro docente de la escuela utiliza de forma empírica los lineamientos normativos tanto en la producción de textos académicos, en el caso de producirlos, como en el desarrollo de proyectos académicos de sus aprendices. El planteamiento del problema descrito nos dirige a ciertas preguntas de investigación: ¿cuántos tipos de lineamientos conocen los profesores 


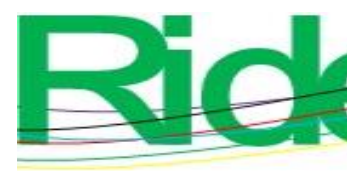
Revista Iberoamericana para la
Investigación y el Desarrollo Educativo
ISSN $2007-7467$

de la Escuela de Nutrición como posibles herramientas a utilizar en la producción de textos académicos? ¿Cuáles serán los lineamientos normativos que más ocupan los profesores en la elaboración de proyectos investigativos? ¿Cuántos tipos de textos académicos conocen los profesores?

La producción de textos académicos en el nivel superior y posgrado resulta tarea ardua pero necesaria en este ámbito de competencia. La calidad de estos documentos es de fundamental interés para las universidades, centros tecnológicos e instituciones de educación superior, puesto que contribuye al avance del conocimiento científico y de la ciencia, lo que beneficia intrínsecamente a la sociedad, además de propiciar la comunicación entre las comunidades científicas afines a las líneas de formación y disciplinas particulares de las licenciaturas de cada estado, región o país.

Asimismo, tomando en cuenta que estudios investigativos han desarrollado proyectos que persiguieron la identificación de los componentes que de alguna forma se involucran en el avance de habilidades estudiantiles que contribuyen a alcanzar una alfabetización académica de calidad, surgió la necesidad de compilar los principios teóricos de este rubro, con el fin de poner documentos solventados y validados al servicio del conocimiento, la ciencia y la sociedad (Mendoza, Escobar y Boza, 2018).

Al redactar textos académicos, el investigador pone a prueba su capacidad comunicativa, su estilo narrativo, descriptivo, argumentativo o explicativo. Sin duda es necesario contar con habilidad, confianza y práctica secuenciada y permanente para realizar esta labor. La capacidad de producir textos académicos muestra parte de la personalidad del productor de estos y revela la capacidad intelectual para comunicarse de manera clara y precisa con la comunidad científica. La pregunta ineludible es: ¿cómo se alcanza dicha habilidad? Y la respuesta no es otra que jtan solo escribiendo! Es decir, la ejecución de la producción de textos genera, a su vez, la práctica necesaria para la adquisición de dicha habilidad (Moreno, Marthe y Rebolledo, 2015). La escritura es una tarea cotidiana en cualquier ámbito del ejercicio profesional, pero cuando se trata de escribir en un rubro profesional específico parece tomar mayores grados de dificultad, mucho más si hablamos del campo de la investigación y su característica producción de tesis, proyectos o artículos. De la misma forma, los profesionales de distintas ramas enfrentarán esta misma dificultad al desarrollar documentos propios en su quehacer laboral (Roldán, 2011). 


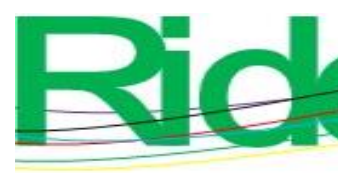

Revista Iberoamericana para la Investigación y el Desarrollo Educativo ISSN $2007-7467$

La escritura asiste los vínculos entre lo que examinan, saben, conciben y repasan los estudiantes. En efecto, proyectos de investigación educativa como el de Flores (2016) indican que los escolares que leen con frecuencia exhiben un mayor pensamiento crítico en su quehacer cotidiano: "En vez de aceptar ideas, conceptos o creencias de forma pasiva, [...] cuestiona, busca problemas interesantes, y a su vez, intenta encontrar argumentos a los mismos" (p. 134). Es así como la lectura, en la mayoría de los horizontes académicos, es indispensable para el imponderable beneficio de los alumnos, así como para la perfección de su ocupación intelectual y epistémica en todas las expresiones del ámbito escolar. Los requerimientos actuales, a raíz de un mundo globalizado, demandan destrezas como el pensamiento crítico, que es forzoso para una lectura razonada y académicamente admisible. La lectura y la escritura originan y sustentan la tendencia crítica en la instrucción de los discentes (Flores, 2016, p. 128)

\section{Materiales y métodos}

El presente estudio es de tipo descriptivo. Se trata de un diseño observacionaltransversal. La muestra u objeto de estudio son los docentes de la Escuela de Nutrición de la Universidad Regional del Sureste. El muestreo que se utilizó fue de tipo probabilístico, aleatorio por estratos, con un cálculo de tamaño de muestra para población finita, con un nivel de confianza o seguridad de $95 \%$, con una precisión de $3 \%$ y ajustando las pérdidas posibles a $24 \%$. Asimismo, el presente estudio es de causalidad con carácter dicotómico, es decir, cuenta con una variable dependiente, conformada por los docentes de la Escuela de Nutrición, y una variable independiente, formulado como "Grado de alfabetización académica en los profesores de la Escuela de Nutrición” (ver tabla 1).

Tabla 1. Representación esquemática de la operacionalización de variables utilizada en el presente proyecto

\begin{tabular}{|c|c|}
\hline Variable dependiente & Variable independiente \\
\hline Profesores de la Escuela de Nutrición & Grado de conocimiento en alfabetización \\
académica
\end{tabular}

Fuente: Elaboración propia 


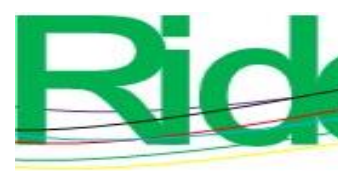

Revista Iberoamericana para la
Investigación y el Desarrollo Educativo ISSN $2007-7467$

\section{Descripción de variables}

En el área biomédica existen requisitos normativos específicos conocidos como normas Vancouver, que son lineamientos para la producción de textos académicos de divulgación, principalmente en revistas científicas del rubro médico. La congregación de estas normas se remonta al año 1978, cuando editores de revistas médicas se reunieron en Canadá para tratar de concertar los lineamientos específicos a seguir en la producción de artículos científicos médicos. Estas pautas o exigencias se anunciaron por primera vez en 1979 con el título de Uniform Requirements for Manuscripts Submitted to Biomedical Journals (Biblioteca de la Universidad de Málaga, 2013).

Si bien el surgimiento de las normas de la American Psychological Association (APA) se dio en el terreno de la psicología, esto no fue un limitante para que se extendieran a otras líneas formativas de las ciencias sociales, las económicas, las ciencias aplicadas, debido a su gran versatilidad en la estructuración de escritos de publicación y de productos académicos de orden docente o estudiantil. Las normas APA son una serie de pautas trazadas para proporcionar una interlocución escrita congruente en divulgaciones académicas, sustancialmente en la introducción de citas y referencias bibliográficas de fuentes de información (Moreno y Castillo, 2019).

Las normas de la Modern Language Association (MLA) surgieron a mediados de los años 80. Se trata de una herramienta indispensable en líneas de investigación del orden social, esencialmente en el área sociocultural, proyectos literarios, lingüísticos y rubros relacionados. Las normas MLA establecen lineamientos de redacción, citación y referencias bibliográficas, principalmente para proyectos de tesis, redacción de libros y artículos de revistas científicas de orden humanístico (Dirección de Sistema de Bibliotecas de la Universidad Finis Terrae, 2018, p. 3).

\section{Descripción del proceso metodológico}

El presente estudio propone las variables anteriormente descritas, las normas Vancouver, APA y MLA, teniendo como finalidad la indagación del grado de conocimiento y uso de estas por parte de los profesores del claustro docente de la Escuela de Nutrición de la Universidad Regional del Sureste. Para ello, se aplicó como instrumento de recolección de datos un cuestionario específico para tal efecto (anexo 1). La vía de aplicación del instrumento fue en línea, con ayuda de la plataforma educativa Edmodo. El análisis 


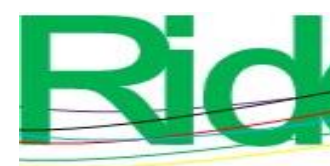

은

Revista Iberoamericana para la Investigación y el Desarrollo Educativo

ISSN 2007 - 7467

estadístico se apoyó del estudio de frecuencias correspondiente, el cual se realizó en los programas estadísticos EPI Info 7.0 y SPSS 22.0. Los resultados y conclusiones fueron canalizados a las áreas de coordinación y dirección de la escuela para la posible elaboración de una antología o manual específico interno de la escuela, el cual tendrá como propósito la unificación de criterios en la producción de textos académicos propios de las diferentes asignaturas.

\section{Resultados}

A partir del cuestionario aplicado al claustro docente de la Escuela de Nutrición, se obtuvieron los siguientes resultados de frecuencias expresados en porcentaje promedio. En cuanto a las preguntas de si conoce y aplica en su labor docente las normas Vancouver, se obtuvo $20 \%$ de promedio de afirmación.

Tabla 2. Preguntas respectivas al conocimiento y aplicación de las normas Vancouver utilizadas en el instrumento de recolección de datos.

\begin{tabular}{|c|c|c|c|c|}
\hline ¿Conoce las & ¿Aplica las & ¿Aplica las & ¿En su quehacer & \% de \\
normas & normas & normas & docente, qué & afirmación \\
Vancouver & Vancouver en la & Vancouver en la & tanto considera & $20 \%$ \\
aplicables a la & producción de & producción de & que hace uso de & \\
producción de & sus textos & textos & las normas & \\
textos & académicos? & académicos de & Vancouver? & \\
académicos? & & sus estudiantes? & & \\
& & & & \\
& & & & \\
& & & & \\
\end{tabular}

Fuente: Elaboración propia

Por su parte, los resultados promedio correspondientes al grado de conocimiento y aplicación de las normas APA, tanto en la producción de textos propios como de los alumnos y de su uso cotidiano en la labor docente, se muestran en la tabla 3. 
Tabla 3. Preguntas respectivas al conocimiento y aplicación de las normas APA utilizadas en el instrumento de recolección de datos.

\begin{tabular}{|c|c|c|c|c|}
\hline ¿Conoce las & ¿Aplica las normas & ¿Aplica las & ¿En su & $\%$ de \\
normas APA & APA a la & normas APA en & quehacer & afirmación \\
aplicables en la & producción de sus & la producción & docente, qué & $75 \%$ \\
producción de & textos académicos? & de textos & tanto \\
textos & & académicos de & considera que \\
académicos? & & sus estudiantes? & hace uso de \\
& & & las normas & \\
& & & APA? & \\
& & & & \\
& & & & \\
& & & & \\
\end{tabular}

Fuente: Elaboración propia

En cuanto a los resultados promedio correspondientes a la aplicación de las normas MLA, tanto en la producción de textos propios como de los alumnos y de su uso cotidiano en la labor docente, se muestran en la tabla 4.

Tabla 4. Preguntas respectivas al conocimiento y aplicación de las normas MLA utilizadas en el instrumento de recolección de datos.

\begin{tabular}{|c|c|c|c|c|}
\hline $\begin{array}{c}\text { ¿Conoce las } \\
\text { normas MLA }\end{array}$ & $\begin{array}{c}\text { ¿Aplica las } \\
\text { normas MLA }\end{array}$ & $\begin{array}{c}\text { ¿Aplica las } \\
\text { normas MLA }\end{array}$ & $\begin{array}{c}\text { ¿En su } \\
\text { quehacer, }\end{array}$ & $\begin{array}{c}\% \text { de } \\
\text { afirmación }\end{array}$ \\
\hline $\begin{array}{l}\text { aplicables en la } \\
\text { producción de } \\
\text { textos } \\
\text { académicos? }\end{array}$ & $\begin{array}{c}\text { en la } \\
\text { producción de } \\
\text { sus textos } \\
\text { académicos? }\end{array}$ & $\begin{array}{c}\text { en la } \\
\text { producción de } \\
\text { textos } \\
\text { académicos de } \\
\text { sus estudiantes? }\end{array}$ & $\begin{array}{c}\text { docente qué } \\
\text { tanto considera } \\
\text { que hace uso de } \\
\text { las normas } \\
\text { MLA? }\end{array}$ & $5 \%$ \\
\hline
\end{tabular}

Fuente: Elaboración propia

Por otra parte, en cuanto a la pregunta relacionada sobre la percepción de uso de las normas Vancouver de forma general por parte de los estudiantes, se obtuvo el siguiente promedio de respuestas positivas por parte de los profesores (ver tabla 5). 


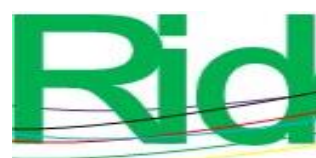

Revista Iberoamericana para la Investigación y el Desarrollo Educativo ISSN 2007 - 7467

Tabla 5. Pregunta del instrumento de recolección de datos la cual estima la percepción de uso de las normas Vancouver

\begin{tabular}{|c|c|}
\hline ¿Bajo su percepción, cómo & \% de afirmación \\
considera que los estudiantes & \\
\cline { 2 - 2 } hacen uso de las normas & $5 \%$ \\
Vancouver en las distintas & \\
asignaturas que cursan? & \\
& \\
\end{tabular}

Fuente: Elaboración propia

De la misma forma, para las normas APA y las MLA se obtuvo un promedio de respuestas positivo respecto a la percepción de uso de estas (ver tablas 6 y 7)

Tabla 6. Pregunta del instrumento de recolección de datos la cual estima la percepción de uso de las normas Vancouver

\begin{tabular}{|c|c|}
\hline ¿Bajo su percepción, cómo & \% de afirmación \\
considera que los estudiantes & \\
\cline { 2 - 2 } hacen uso de las normas APA & $92 \%$ \\
en las distintas asignaturas que & \\
cursan? & \\
& \\
\end{tabular}

Fuente: Elaboración propia 
Tabla 7. Pregunta del instrumento de recolección de datos la cual estima la percepción de uso de las normas Vancouver

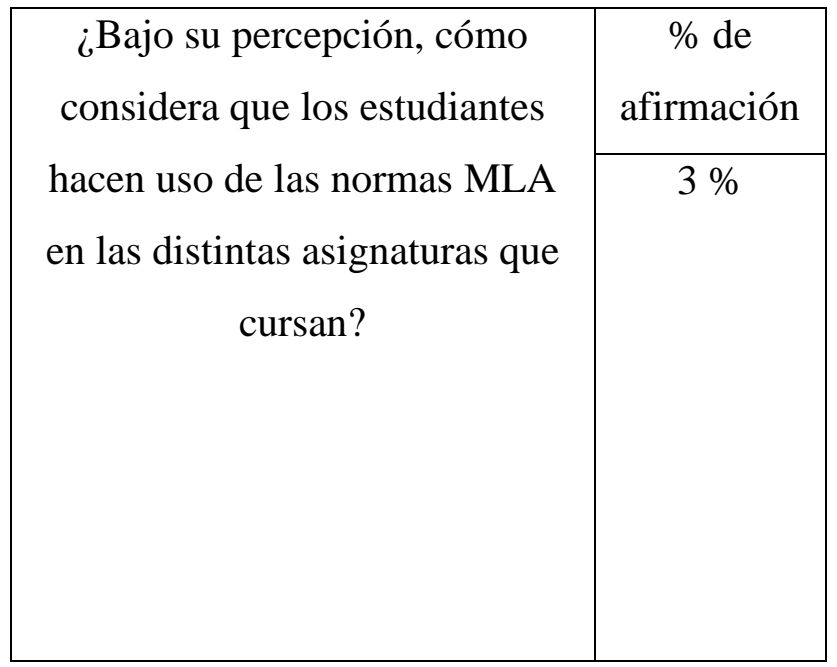

Fuente: Elaboración propia

Finalmente, para las preguntas relacionadas con el conocimiento y uso de los diferentes tipos de textos académicos, se obtuvo $3 \%$ de frecuencia de afirmaciones positivas promedio para las normas Vancouver.

Tabla 8. Preguntas referentes al conocimiento y aplicación de las normas Vancouver en la producción de textos académicos

\begin{tabular}{|c|c|c|}
\hline $\begin{array}{c}\text { ¿Conoce los } \\
\text { diferentes tipos }\end{array}$ & $\begin{array}{c}\text { ¿Si conoce los } \\
\text { diferentes tipos de }\end{array}$ & $\begin{array}{c}\% \text { de } \\
\text { afirmación }\end{array}$ \\
\hline de textos & textos, qué tanto & $3 \%$ \\
\hline académicos: & uso hace de ellos en & \\
\hline ensayos, & la producción de & \\
\hline relatorías, & trabajos & \\
\hline monografías, & académicos de sus & \\
\hline etc.? & estudiantes & \\
\hline & apegándose a las & \\
\hline & normas Vancouver? & \\
\hline
\end{tabular}

Fuente: Elaboración propia

Y para las normas APA y MLA, para este mismo rubro de preguntas relacionadas con el conocimiento y uso de los diferentes tipos de textos académicos, se obtuvieron los siguientes resultados (ver tablas 9 y 10). 
Tabla 9. Preguntas referentes al conocimiento y aplicación de las normas APA en la producción de textos académicos

\begin{tabular}{|c|c|c|}
\hline ¿Conoce los & ¿Si conoce los & $\%$ de \\
diferentes tipos & diferentes tipos de & afirmación \\
\cline { 3 - 3 } de textos & textos, qué tanto & $95 \%$ \\
académicos: & uso hace de ellos en & \\
ensayos, & la producción de & \\
relatorías, & trabajos & \\
monografías, & académicos de sus & \\
etc.? & estudiantes & \\
& apegándose a las & normas APA? \\
\hline
\end{tabular}

Fuente: Elaboración propia

Tabla 10. Preguntas referentes al conocimiento y aplicación de las normas MLA en la producción de textos académicos

\begin{tabular}{|c|c|c|}
\hline ¿Conoce los diferentes & ¿Si conoce los & $\%$ de afirmación \\
tipos de textos & diferentes tipos de & \\
\cline { 3 - 3 } académicos: ensayos, & textos, qué tanto uso & $2 \%$ \\
relatorías, monografías, & hace de ellos en la & \\
etc.? & producción de trabajos & \\
& académicos de sus & \\
& estudiantes apegándose & \\
& a las normas MLA? & \\
\hline
\end{tabular}

Fuente: Elaboración propia 


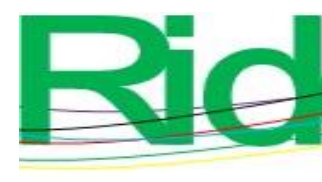

Revista Iberoamericana para la Investigación y el Desarrollo Educativo ISSN $2007-7467$

\section{Discusión}

Los profesores de educación superior tienen la enmienda de producir textos académicos como medio de difusión de ideas. Lo anterior con el fin de dar a conocer contenidos de relevancia propia de los diferentes perfiles profesionales. Asimismo, cuando se deja como tarea la realización de un trabajo escrito a los estudiantes, es necesario establecer los lineamientos pertinentes y claros en la elaboración de este (Roa, 2014, pp. 46). La producción de textos académicos, ya sea de parte del profesor o del estudiante, implica retomar temas tan básicos en la educación escolar como son la escritura y la lectura, ya mencionados en detalle en la introducción del presente proyecto.

Establecer lineamientos claros y precisos tiene que ser una dinámica que con la práctica se convierta en un ejercicio cotidiano en el quehacer universitario (Chico, 2016, p. 11). Un trabajo de investigación, es decir, un texto académico (paper) debe tener características básicas para poder ser considerado de buen nivel científico. Dentro de estas características, se tiene que el contenido debe ser actual, novedoso, interesante, original y relevante. El grado de descripción debe ser pertinente, con una redacción fluida y amable para el lector, además de utilizar una terminología adecuada, aunque siempre comprensible para cualquier tipo de comunidad (Perona, 2005).

A pesar de que las normas Vancouver son regularmente utilizadas dentro del ámbito de los profesionales de la salud en proyectos educativos, los resultados del presente estudio arrojan un bajo uso de ellas, tanto en la producción de textos académicos que realizan los estudiantes como los profesores. De forma contraria, se observa un alto uso de las normas APA. La explicación posible del fenómeno observado radica en la hegemonía que desde hace décadas ha establecido la APA, situación posible de observar desde el campo académico hasta el área comercial, por la cantidad de instituciones de educación superior que utilizan estos lineamientos como ejes rectores de su producción de textos académicos, así como por el gran número de cursos de actualización que son ofrecidos por esta asociación.

En lo que se refiere a las normas MLA, se observa un muy bajo uso y conocimiento dentro de la comunidad estudiantil de la Escuela de Nutrición de la Universidad Regional del Sureste. La explicación posible estriba en la baja cantidad de asignaturas de perfil sociológico con la que cuenta el programa de estudios de la licenciatura. No obstante, se tendría que revalorar su uso, puesto que la licenciatura en Nutrición cuenta con líneas de formación 


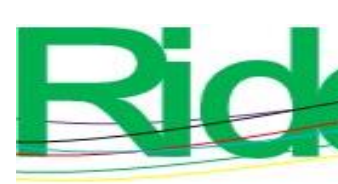

Revista Iberoamericana para la
Investigación y el Desarrollo Educativo
ISSN $2007-7467$

diversas y una de ellas es la de nutrición poblacional, perfil que aborda contenidos netamente sociológicos.

Finalmente, uno de los hallazgos que pone de manifiesto el presente estudio es el alto porcentaje de profesores del claustro docente de la Escuela de Nutrición de la Universidad Regional del Sureste que conoce los diferentes tipos de textos académicos (ensayos, monografías, relatorías, etc.). Seguramente porque son utilizados como instrumentos didácticos para abordar contenidos pertenecientes a los diferentes programas de estudio de las diversas asignaturas, motivo por el cual se considera necesario establecer un manual que funcione como guía dentro de la Escuela de Nutrición y que aborde los tres tipos de normatividades tratadas en el presente estudio (Vancouver, APA y MLA) con el fin de unificar criterios referentes a la elaboración de textos académicos.

\section{Conclusiones}

Respecto al conocimiento y aplicación de las normas Vancouver, menos de una cuarta parte de los profesores del claustro docente de la Escuela de la Nutrición las identifica con claridad y, por extensión, las utiliza con poca frecuencia. La explicación de estos resultados tal vez sea debido a que un porcentaje de profesores no se encuentra en la necesidad de elaborar artículos de investigación de autoría personal, así como debido al hecho de que los contenidos de su asignatura no requieren la elaboración de documentos formales dentro del área de la salud.

Lo contrario sucede con las normas APA, ya que por lo menos tres cuartas partes de los profesores de la escuela las conocen; las aplican durante la producción de textos académicos personales y en los demandados a sus alumnos. Así, se percibe un mayor dominio académico por parte de estas normas. La explicación posible es que existe una mayor cantidad integrados a asociaciones de investigación y organismos formales de producción de textos académicos.

En conclusión, tenemos que el conocimiento y aplicación de las normas MLA cuentan con un muy bajo porcentaje de uso dentro de la Escuela de Nutrición. Lo más pertinente de deducir de dichos resultados es que este tipo de normas pertenecen al área social propiamente, por lo que solo tendrían uso en aquellas asignaturas que sus contenidos cuenten con este tipo de enfoque, las cuales forman parte de un pequeño porcentaje en el plan de estudios de la licenciatura en Nutrición. 


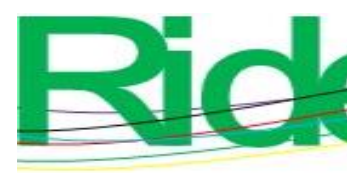

Revista Iberoamericana para la
Investigación y el Desarrollo Educativo
ISSN $2007-7467$

Por otra parte, la percepción de los profesores sobre el uso que hacen los estudiantes en sus distintas asignaturas de las normas Vancouver, APA y MLA no varió en gran medida de los resultados anteriormente descritos, puesto que el mayor porcentaje de frecuencias afirmativas de los docentes fue para las normas APA, continuando en orden decendiente las Vancouver y las MLA. Sin duda las normas de la APA cuentan con un mayor dominio dentro del campo de la investigación y producción de textos académicos, esto debido, como ya se mencionó con anterioridad, a la mayor estructuración, aplicación y difusión que le dan a su normatividad.

Finalmente, se concluye que los profesores de la Escuela de Nutrición de la Universidad Regional del Sureste conocen los diferentes tipos de textos académicos (ensayos, relatorías y las monografías). De la misma forma, la mayor normativa aplicada en la elaboración de trabajos escolares por parte de sus estudiantes es la de la APA. Con base en todas las conclusiones anteriores, se propone para el presente proyecto la elaboración de un manual normativo de la producción de textos académicos interno de la propia escuela, que brinde homogeneidad a los documentos que producen los estudiantes en las diferentes asignaturas que cursan a lo largo de su carrera.

\section{Futuras líneas de investigación}

El presente proyecto de investigación abre una línea de investigación dirigida al análisis más profundo de las diferentes convergencias y divergencias que existen entre los diferentes tipos de normatividades, APA, Vancouver y MLA, principalmente. Es decir, que un proceso investigativo futuro tendría que analizar cuáles lineamientos de las tres normatividades se entrelazan, así como las diferencias sustanciales que presentan.

Todo lo anterior con la finalidad de generar un manual normativo de la producción de textos académicos, tanto de uso interno en la Escuela de Nutrición de la Universidad Regional del Sureste de la ciudad de Oaxaca como para que sirva a la comunidad científica de otras instituciones. Este manual normativo de la producción de textos académicos deberá contemplar los resultados obtenidos del proceso investigativo respectivo, a fin de amalgamar los lineamientos más sobresalientes y funcionales, a modo de hacerlo versátil y aplicable a los diferentes tipos de asignaturas. 


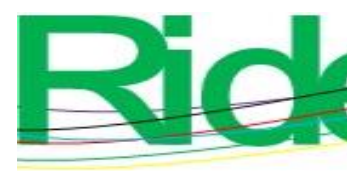

Revista Iberoamericana para la
Investigación y el Desarrollo Educativo
ISSN $2007-7467$

Esta idea ecléctica de la recopilación de información de los lineamientos de las normatividades APA, Vancouver y MLA deberá, de la misma forma, cuidar los aspectos de mayor uso y aplicabilidad global de estos en la producción de textos académicos o, en su caso, respetar la estructura del documento a generar, es decir, que al proceso investigativo futuro mencionado le corresponderá analizar los diferentes tipos de documentos investigativos que existen, indagando su estructura y tipos de párrafos que utilizan, además de no dejar de visualizar la línea de formación a la que pertenecen, esto último concerniente a líneas de formación con las que cuenta el plan de estudios de la Escuela de Nutrición, a saber, del área médica, área social y antropológica, además del área técnica específica de la disciplina.

Finalmente, se puede percibir que futuras investigaciones servirán de sustento para aumentar el grado de conocimiento de los profesores de educación superior y con esto favorecer la elaboración de un mejor producto y calidad de textos académicos.

\section{Referencias}

Andrade, M. (2009). La escritura y los universitarios. Cundinamarca: Universidad Colegio Mayor de Cundinamarca, Colombia.

Barreto, M. (2014). La alfabetización académica en la formación universitaria. (Trabajo especial de grado). Universidad Militar Nueva Granada, Bogotá.

Biblioteca de la Universidad de Málaga. (2013). Normas de Vancouver. Guía breve. Málaga, España: Universidad de Málaga.

Carlino, P. (2017). Dos variantes de la alfabetización académica cuando se entrelazan la lectura y la escritura en las materias. Signo y Pensamiento, 36(71), 16-32. Recuperado de https://www.redalyc.org/pdf/860/86054913001.pdf.

Chico, J. (2016). Manual de textos académicos 2016. Guanajuato: Instituto Renacimiento.

Dirección de Sistema de Bibliotecas de la Universidad Finis Terrae. (2018). Normas MLA.

Chile: Universidad Finis Terrae. Recuperado de https://uft.cl/images/biblioteca/descargables/norma-mla-2018.pdf.

Fernández, E. (2017). Una mirada a los desafíos de la educación superior en México.

Innovación Educativa, 17(74), 183-207. Recuperado de http://www.scielo.org.mx/pdf/ie/v17n74/1665-2673-ie-17-74-00183.pdf. 
Roa, M. A. (2014). Guía para la elaboración y registro de textos o trabajos académicos. Veracruz: Instituto Tecnológico de Minatitlán.

Roldán, C. (2011). Mirar la escritura en la educación superior como un prisma. Revista Iberoamericana de Educación, 55(3), 1-13. Recuperado de https://rieoei.org/historico/deloslectores/4010Roldan.pdf.

Rué, D. (2015). El desarrollo profesional docente en educación superior: agenda, referentes y propuestas para su adopción. Revista de Docencia Universitaria, 13(extraordinario), 217-236.

\section{Anexos}

\section{Anexo 1. Instrumentos de recolección de datos}

El cuestionario de recolección de datos contó con las siguientes fichas de identificación:

Tabla 11. Ficha de identificación de datos generales del cuestionario de recolección de datos.

\begin{tabular}{|l|l|}
\hline \multicolumn{2}{|c|}{ Ficha de identificación } \\
\hline Nombre: & \\
\hline Asignatura (s) que imparte: & \\
\hline $\begin{array}{l}\text { Número de grupos a los que le } \\
\text { imparte clase: }\end{array}$ & \\
\hline
\end{tabular}

Fuente: Elaboración propia 
Tabla 12. Ficha de identificación de preguntas referentes a las normas Vancouver.

\begin{tabular}{|c|c|c|c|c|}
\hline $\begin{array}{c}\text { Contesta según tu criterio de } \\
\text { aplicación }\end{array}$ & \multicolumn{4}{|c|}{$\checkmark$ Marque con una $X$} \\
\hline $\begin{array}{lcr}\text { ¿Conoce } & \text { las } & \text { normas } \\
\text { Vancouver } & \text { aplicables a la } \\
\text { producción } & \text { de } & \text { textos } \\
\text { académicos? } & & \\
\end{array}$ & \multicolumn{2}{|c|}{ Sí } & \multicolumn{2}{|c|}{ no } \\
\hline $\begin{array}{l}\text { ¿Aplica las normas } \\
\text { Vancouver en la producción } \\
\text { de sus textos académicos? }\end{array}$ & \multicolumn{2}{|c|}{ Sí } & \multicolumn{2}{|c|}{ No } \\
\hline $\begin{array}{l}\text { ¿Aplica las normas } \\
\text { Vancouver en la producción } \\
\text { de textos académicos de sus } \\
\text { estudiantes? }\end{array}$ & \multicolumn{2}{|c|}{ Sí } & \multicolumn{2}{|c|}{ No } \\
\hline $\begin{array}{l}\text { ¿En su quehacer docente, qué } \\
\text { tanto considera que hace uso } \\
\text { de las normas Vancouver? }\end{array}$ & Bastante & Regular & Poco & Nada \\
\hline $\begin{array}{l}\text { ¿Bajo su percepción, cómo } \\
\text { considera que los estudiantes } \\
\text { hacen uso de las normas } \\
\text { Vancouver en las distintas } \\
\text { asignaturas que cursan? }\end{array}$ & Bastante & Regular & Poco & Nada \\
\hline $\begin{array}{l}\text { ¿Conoce los diferentes tipos } \\
\text { de textos académicos: } \\
\text { ensayos, relatorías, } \\
\text { monografías, etc.? }\end{array}$ & \multicolumn{2}{|c|}{ Sí } & \multicolumn{2}{|c|}{ No } \\
\hline $\begin{array}{l}\text { ¿Si conoce los diferentes tipos } \\
\text { de textos, qué tanto uso hace } \\
\text { de ellos en la producción de } \\
\text { trabajos académicos de sus } \\
\text { estudiantes apegándose a las } \\
\text { normas Vancouver? }\end{array}$ & Bastante & Regular & Poco & Nada \\
\hline
\end{tabular}


Fuente: Elaboración propia

Tabla 13. Ficha de identificación de preguntas referentes a las normas APA.

\begin{tabular}{|c|c|c|c|c|}
\hline $\begin{array}{c}\text { Contesta según tu criterio de } \\
\text { aplicación }\end{array}$ & \multicolumn{4}{|c|}{ Marque con una $X$} \\
\hline $\begin{array}{l}\text { ¿Conoce las normas APA } \\
\text { aplicables a la producción de } \\
\text { textos académicos? }\end{array}$ & \multicolumn{2}{|c|}{ Sí } & \multicolumn{2}{|c|}{ No } \\
\hline $\begin{array}{l}\text { ¿Aplica las normas APA en la } \\
\text { producción de sus textos } \\
\text { académicos? }\end{array}$ & \multicolumn{2}{|c|}{ Sí } & \multicolumn{2}{|c|}{ No } \\
\hline 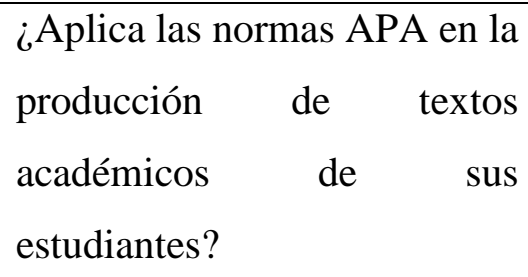 & \multicolumn{2}{|c|}{ Sí } & \multicolumn{2}{|c|}{ No } \\
\hline $\begin{array}{l}\text { ¿En su quehacer docente, qué } \\
\text { tanto considera que hace uso } \\
\text { de las normas APA? }\end{array}$ & Bastante & Regular & Poco & Nada \\
\hline $\begin{array}{l}\text { ¿Bajo su percepción, cómo } \\
\text { considera que los estudiantes } \\
\text { hacen uso de las normas APA } \\
\text { en las distintas asignaturas } \\
\text { que cursan? }\end{array}$ & Bastante & Regular & Poco & Nada \\
\hline $\begin{array}{l}\text { ¿Conoce los diferentes tipos } \\
\text { de textos académicos: } \\
\text { ensayos, relatorías, } \\
\text { monografías, etc.? }\end{array}$ & \multicolumn{2}{|c|}{ Sí } & \multicolumn{2}{|c|}{ No } \\
\hline $\begin{array}{l}\text { ¿Si conoce los diferentes tipos } \\
\text { de textos, qué tanto uso hace } \\
\text { de ellos en la producción de } \\
\text { trabajos académicos de sus }\end{array}$ & Bastante & Regular & Poco & Nada \\
\hline
\end{tabular}




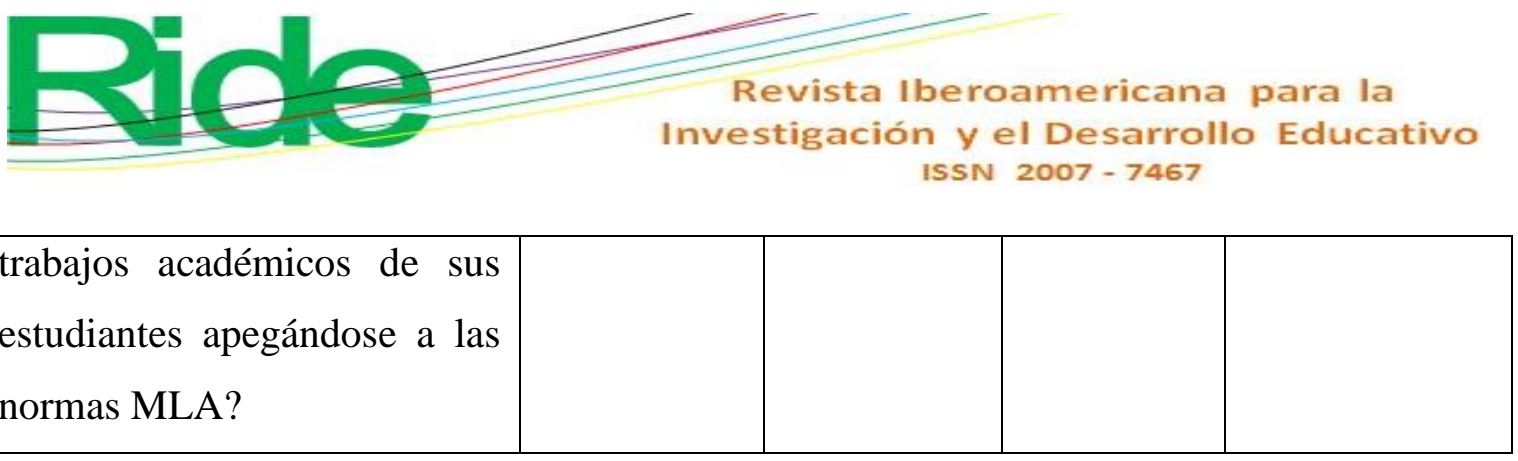

Fuente: Elaboración propia

Vol. 12, Núm. 23 Julio - Diciembre 2021, e254 